\title{
Application of the Chinese Aircraft-shaped Sleeve system in the treatment of tibial shaft fractures using a suprapatellar approach for tibial intramedullary nailing: a randomised controlled trial
}

Ke Lu' ${ }^{1,2}$, Ting-ting Zhou ${ }^{3}$, Yi-jun Gao ${ }^{2}$, Hong-zhen Wang ${ }^{2}$, Zhi-qiang Wu'², Yuan Wang ${ }^{2}$, Dong-gui Zheng ${ }^{4}$ and Qi-rong Dong ${ }^{1 *}$ (D)

\begin{abstract}
Background: The use of the suprapatellar approach for intramedullary nailing has recently become popular for the treatment of tibial fractures. This study aimed to investigate the effectiveness of using the Chinese Aircraft-shaped Sleeve (CASS) system on the clinical outcomes of tibial intermedullary nailing using a suprapatellar approach for the treatment of tibial fractures in a cohort of adult Chinese patients.

Methods: Magnetic resonance images (MRI) of the knee joints of 212 healthy adults with normal lower limb function from May 2011 to May 2015 were obtained from a level I Chinese regional trauma centre. Sixty inpatients at the same trauma centre who underwent treatment for tibial shaft fractures from June 2015 to June 2018 were enrolled. Tibial intramedullary nailing fixation of tibial shaft fractures via the suprapatellar approach was performed with either a CASS system or a conventional sleeve. The measurements of patellofemoral joint anatomy, the surgical time, the assessments of the patellofemoral joint cartilage conditions, and information of residual debris based on arthroscopic analysis were subsequently collected.

Results: The mean patellar angle (PA), sulcus angle (SA), sulcus width (SW) and sulcus depth (SD) were $135.40 \pm 6.20^{\circ}, 142.37 \pm 5.33^{\circ}, 33.37 \pm 2.73 \mathrm{~mm}$, and $4.29 \pm 0.63 \mathrm{~mm}$, respectively. The surgical time until entry reaming commencement and the irrigation time were significantly lower in the CASS group $(P<0.001)$. The difference in cartilage damage rate between groups was statistically significant $(P=0.031)$; the difference in residual debris conditions was not statistically significant $(P=0.1967)$.
\end{abstract}

Conclusion: The use of the CASS system could improve clinical outcomes of intramedullary nailing via suprapatellar approach for patients with a small patellofemoral joint space.

Keywords: Suprapatellar approach, Tibial fracture, Intramedullary nailing, Sleeve, Magnetic resonance, Patellofemoral joint

\footnotetext{
* Correspondence: dongqirong@suda.edu.cn

Ke Lu, Ting-ting Zhou, Yi-jun Gao and Hong-zhen Wang are co-first authors.

Ke Lu, Ting-ting Zhou, Yi-jun Gao and Hong-zhen Wang contributed equally to this work.

${ }^{1}$ Department of Orthopaedics, the Second Affiliated Hospital of Soochow

University, 1055 Sanxiang Road, Suzhou 215004, Jiangsu, China

Full list of author information is available at the end of the article
}

(c) The Author(s). 2018 Open Access This article is distributed under the terms of the Creative Commons Attribution 4.0 International License (http://creativecommons.org/licenses/by/4.0/), which permits unrestricted use, distribution, and reproduction in any medium, provided you give appropriate credit to the original author(s) and the source, provide a link to the Creative Commons license, and indicate if changes were made. The Creative Commons Public Domain Dedication waiver (http://creativecommons.org/publicdomain/zero/1.0/) applies to the data made available in this article, unless otherwise stated. 


\section{Background}

Tibial fractures are one of the most common long bone fractures in the human body. Intramedullary nailing (IMN) has become the preferred treatment choice for tibial fractures, particularly those of the shaft, as it is minimally invasive and ensures high fixation strength, which allows patients to return to routine activities immediately after surgery [1]. The traditional surgical approach for IMN begins with an incision below the patella (in the middle or along the patellar ligament), with an insertion of the nail with the knee joint flexed to at least $90^{\circ}$. In 1996, Tornetta and Collins [2] first reported a suprapatellar approach, performed with the knee in a semi-extended position (in about $15^{\circ}$ of flexion), using a mid-line incision, from the superior patellar pole to the tibial tubercle, followed by the creation of a slit through two-thirds of the medial patellar retinaculum to sublux the patella.

Although advances have been made to improve the suprapatellar approach, several technical issues remain. Foremost, existing tools are designed for Western populations and, therefore, may not be appropriate for all other populations. As an example, individuals of Chinese ethnicity tend to be smaller than Westerners and, as such, the patellofemoral joint space is relatively smaller, resulting in greater resistance after incision of the quadriceps and insertion of the outer protective sleeve, compared to the original procedure developed in Westerners. The small patellofemoral joint space also increases the risk of iatrogenic injury to the intra-articular structure, as well as making placement of the guide wire more difficult, which increases operative time. Second, the original protective sleeve used in the original suprapatellar IMN approach is V-shaped, which is a poor match for Chinese individuals. Due to this shape mismatch, when the pressure on the patellofemoral joint is high, the patella slides to one side and the sleeve to the opposite side (Fig. 1), with this instability increasing the difficulty of accurately insert the IM nail. Finally, a large amount of debris is generated during surgical access and reaming, requiring a thorough irrigation prior to incision closure. Ensuring debris evacuation from the joint cavity has become a difficult problem that needs to be addressed. In view of these difficult issues associated with the performance of a suprapatellar approach of IMN among patients with a relatively small patellofemoral joint space, we present in this study a newly designed protective sleeve system that is specifically based on the anatomical parameters of patellofemoral joints in Chinese individuals and describe appropriate modifications to the conventional surgical procedures. Our aim, specifically, was to investigate the effectiveness of the sleeve system in the treatment of tibial shaft fractures using a suprapatellar approach for tibial IMN.

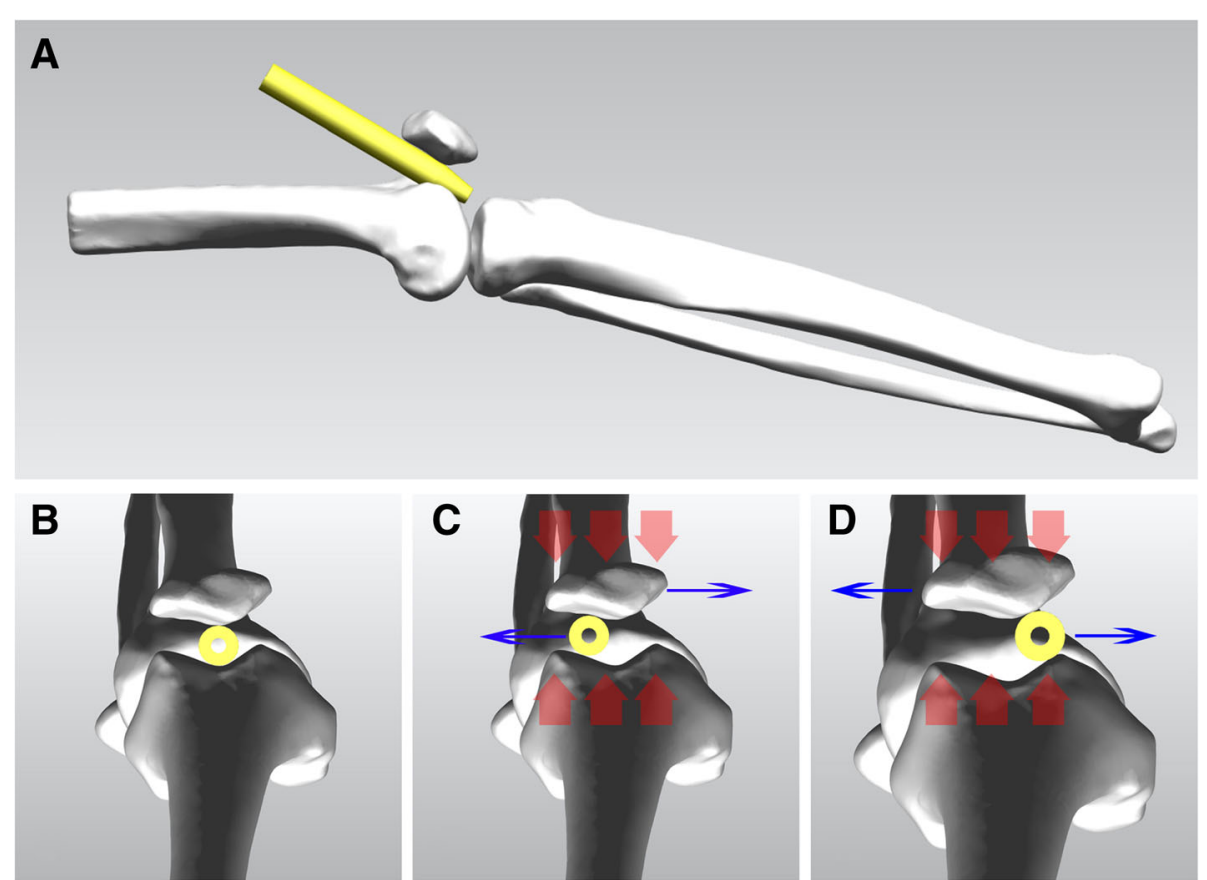

Fig. 1 Instability in the position of the conventional sleeve during intramedullary nailing. $\mathbf{a}$, b The conventional sleeve must be located in the central area of the patellofemoral joint, as viewed from the lateral and axial positions. $\mathbf{c}$, $\mathbf{d}$ The conventional sleeve is round, such that when the pressure on the patellofemoral joint (red arrow) is high, with the patella slides to one side (blue arrow) and the sleeve to the opposite side (blue arrow) 


\section{Methods}

\section{Anatomical parameters of the Chinese Aircraft-shaped} Sleeve (CASS) system

The study was approved by our institutional review board and ethics committee and all participants provided informed consent.

Magnetic resonance images (MRI) of the knee joints of 212 healthy adults with normal lower limb function were obtained from May 2011 to May 2015 from a level I Chinese regional trauma centre. The exclusion criteria were as follows: previous history of knee surgery, trauma, space-occupying lesions in the knee joint and surrounding areas, patellar softening, sulcus dysplasia, synovial hyperplasia, and severe fluid accumulation in the joints. Of the 212 patients, 101 were men and 111 were women, and the age range was $18-73$ years (mean 41 years). In total, there were 106 left knees and 106 right knees. The MRIs were obtained using a 1.5-T system (Philips 1.5-T Achieva, Philips, Netherlands) with patients in the supine position with full knee extension. Axial T2-weighted (TR/TE: shortest/100, matrix 256×192, FOV $160 \times 160$, slice thickness $3 \mathrm{~mm}$ ) sequences were obtained. MRIs were analysed on a workstation (Centricity RIS CE V3.0, General electric medical system (China) Co., Ltd., USA) by one radiologist, and measurements were made using electronic callipers.

The patellar angle (PA) was measured on the axial image of the knee where the patella was the widest. The sulcus angle (SA), sulcus width (SW), and sulcus depth (SD) were measured on axial images of the knee where the transepicondylar axis was fully displayed on the first level from the proximal to the distal ends (Fig. 2).

\section{Application of the CASS system}

Sixty inpatients who underwent treatment for a tibial shaft fracture at our centre, between June 2015 and June 2018, were enrolled. The selection criteria were as follows: (1) tibial shaft fractures (OTA type 42), (2) achievement of mature bone, and (3) the use of the Expert Tibial Nail system (ETN; DePuy Synthes, USA), with a suprapatellar approach. The exclusion criteria were as follows: (1) knee injury or stiffness, (2) patella baja, (3) patellar ligament calcification, (4) existing vascular nerve injury, (5) soft tissue injury around the
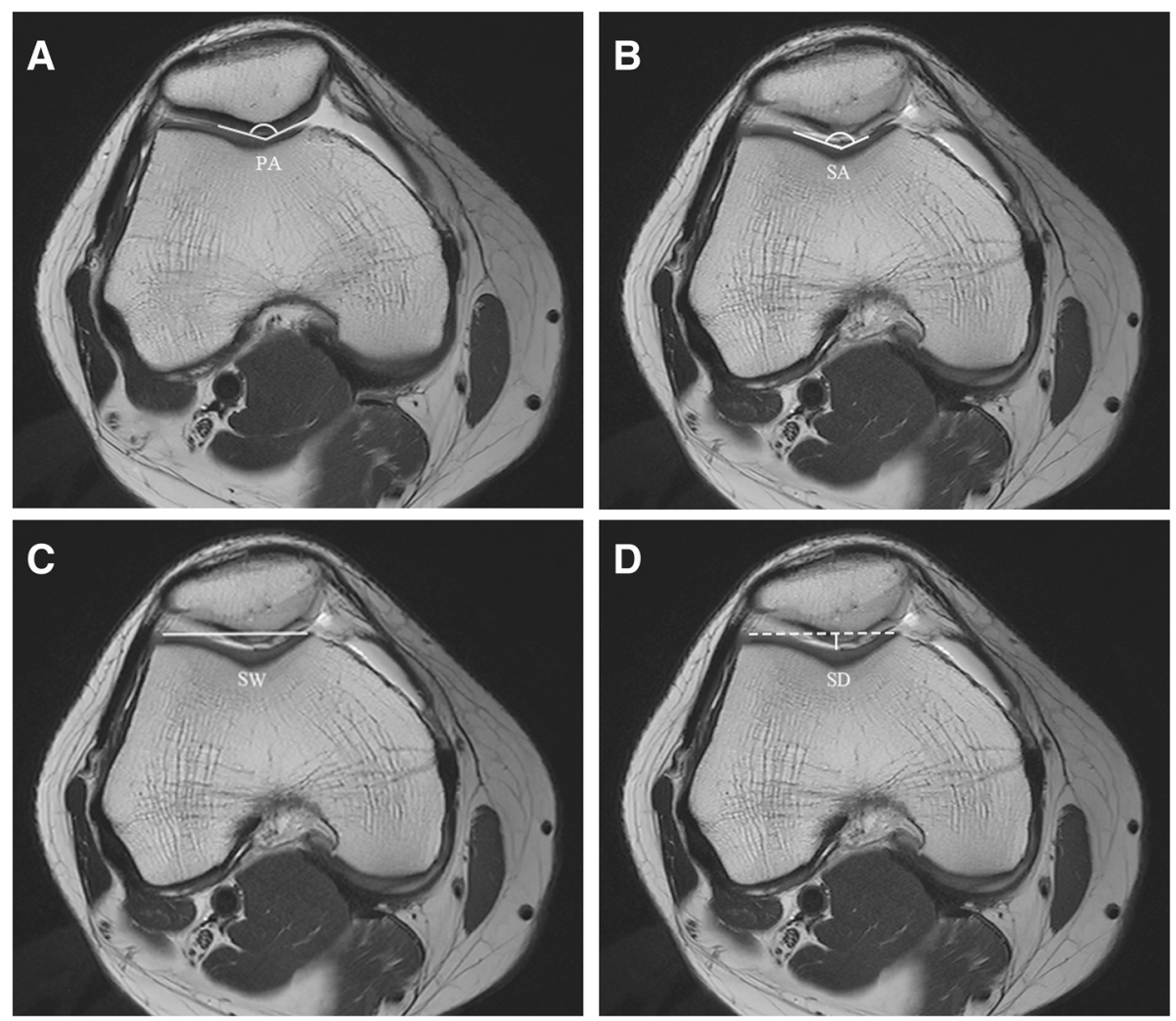

Fig. 2 The following anatomical parameters of the patellofemoral joint (PFJ) anatomy were measured on magnetic resonance images: a Patella angle (PA): for the angle where the medial and lateral cartilage surfaces of the patella meet. b Sulcus angle (SA): the angle between the medial and lateral cartilage surfaces of the sulcus. c Sulcus width (SW): the length of the line connecting the most prominent points of the medial and lateral cartilages inside the trochlear groove. $\mathbf{d}$ Sulcus depth (SD): the length of the perpendicular line from the deepest point between the cartilage of the trochlear groove cartilage and the SW 
incision area, and (6) metabolic or pathological bone diseases. After patients were enrolled in the study, each received a sealed envelope containing a randomised number, generated using SAS 9.2 (SAS Institute, Inc., Cary, NC, USA) based on the order of admission, for allocation to either the CASS group or the conventional sleeve group, in a 1:1 ratio.

The general characteristics of the patient group were as follows: 33 men and 27 women, with an age range of 22 to 69 years. There were no significant differences in baseline conditions between the CASS and conventional sleeve group. All procedures were performed by one senior surgeon. Due to the specific nature of the intervention methods, double-blinding could not be achieved between the surgeon and patients. Data processing, statistical analysis, and evaluations were performed by independent professionals who had no knowledge of group assignment.

\section{Sample size}

Based on preliminary tests and a previous study [3], we initially defined the primary outcome as the rate of cartilage damage, with an estimated rate of 0.03 in the treatment group (CASS group) and 0.28 in the control group (conventional sleeve group). The ratio between the sample sizes of the two groups was 1:1. Using an $\alpha$ value of 0.05 and $\beta$ value of 0.2 (power of 0.80 ), the minimum requirement, based on the SAS sample size calculator procedure [4], was 30 patients per group.

\section{Surgical treatment}

Patients in the CASS group underwent IMN using the original suprapatellar ETN system but with the CASS, which was designed based on the anatomical parameters of the patellofemoral joints measured from the MRI of the sample of 212 healthy individuals, and 3D printed (Fig. 3). The CASS was fabricated using thermoplastic polyurethane (TU), which has a stiffness of 42D-64D. The surgical procedure includes the following (Figs. 4 and 5): preoperative positioning of the patient in the supine position, with a knee pillow under the popliteal fossa of the affected limb to position the knee joint in a semi-extended position (flexion of $10^{\circ}-20^{\circ}$ ). A longitudinal skin incision, of approximately $2-4 \mathrm{~cm}$, was made, $1.5 \mathrm{~cm}$ above the superior patellar pole. The subcutaneous soft tissue was also incised. Deep separation was performed, with the quadriceps tendon being cut and the joint capsule above the patella opened further. An index finger was used to reach the joint capsule for preliminary separation of soft tissue. The patella was revealed anteriorly. The protective sleeve device was then placed, before placement of the guide wire. To address the shape mismatch in the CASS group, the positioning sleeved (aircraft cabin) was custom-designed based on

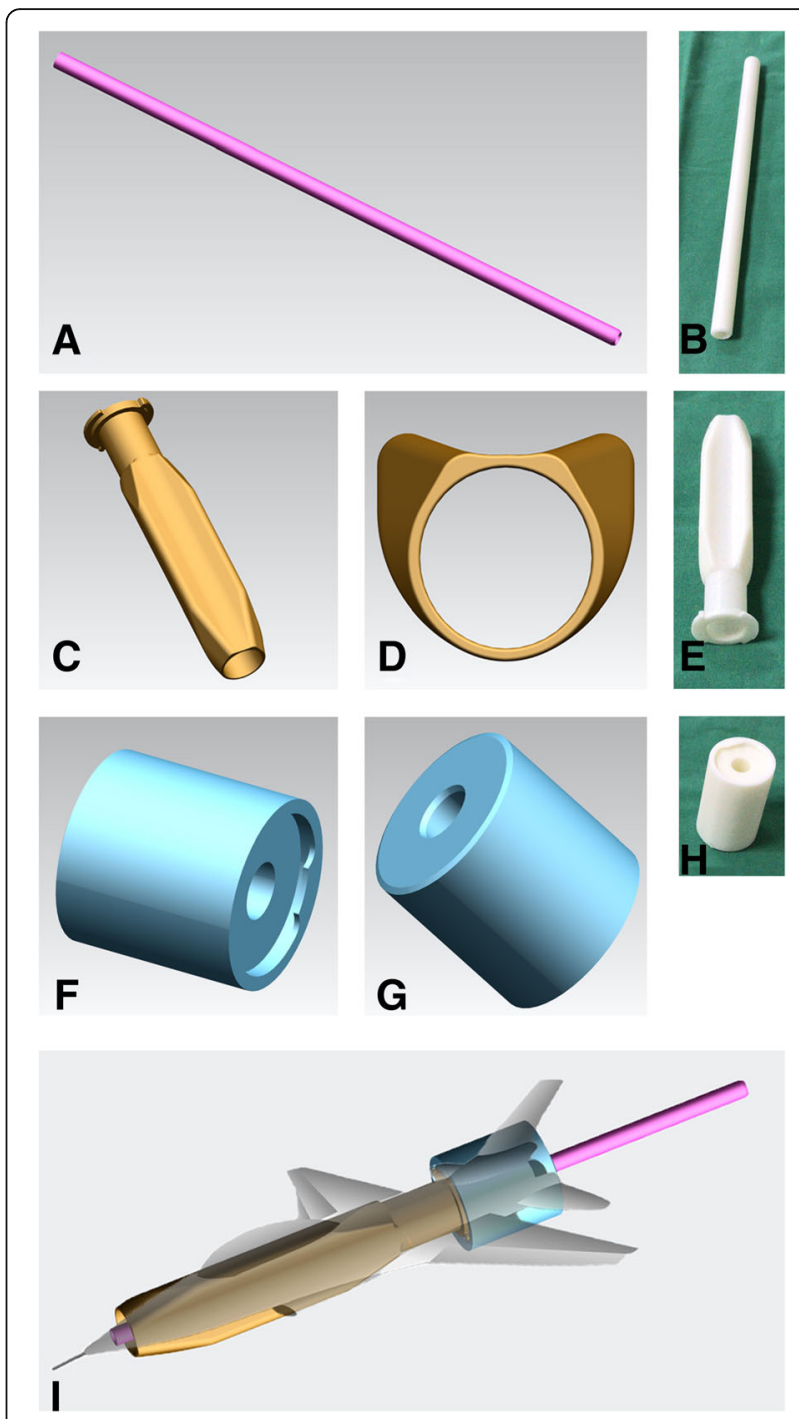

Fig. 3 The Chinese Aircraft-shaped Sleeve system: a, b Positioning of the protective sleeve for the guide-wire-first method (aircraft cabin). c-e Outer protective sleeve (aircraft wings). $\mathbf{f}-\mathbf{h}$ Connector (aircraft tail). $\mathbf{i}$ When the three components are combined into one image, that shape of aircraft sleeve is obtained

the average knee parameters for Chinese individuals, using a translucent material. After opening the joint capsule, the positioning sleeve was gently inserted into the femoral sulcus, up to the tibial guide wire insertion point, and a 3.2-mm guide wire was drilled into it. An intraoperative radiograph was obtained to ensure optimal positioning of the insertion and position of the guide wire. The use of the compact, translucent positioning sleeve facilitated manoeuvring of the guide wire within the patellofemoral joint for accurate positioning. The positioning sleeve was removed, and the sleeve system was inserted (including the handle, inner protective sleeve, and centring sleeve) along the guide wire. The inner layer of the sleeve body has a hollow cylindrical 


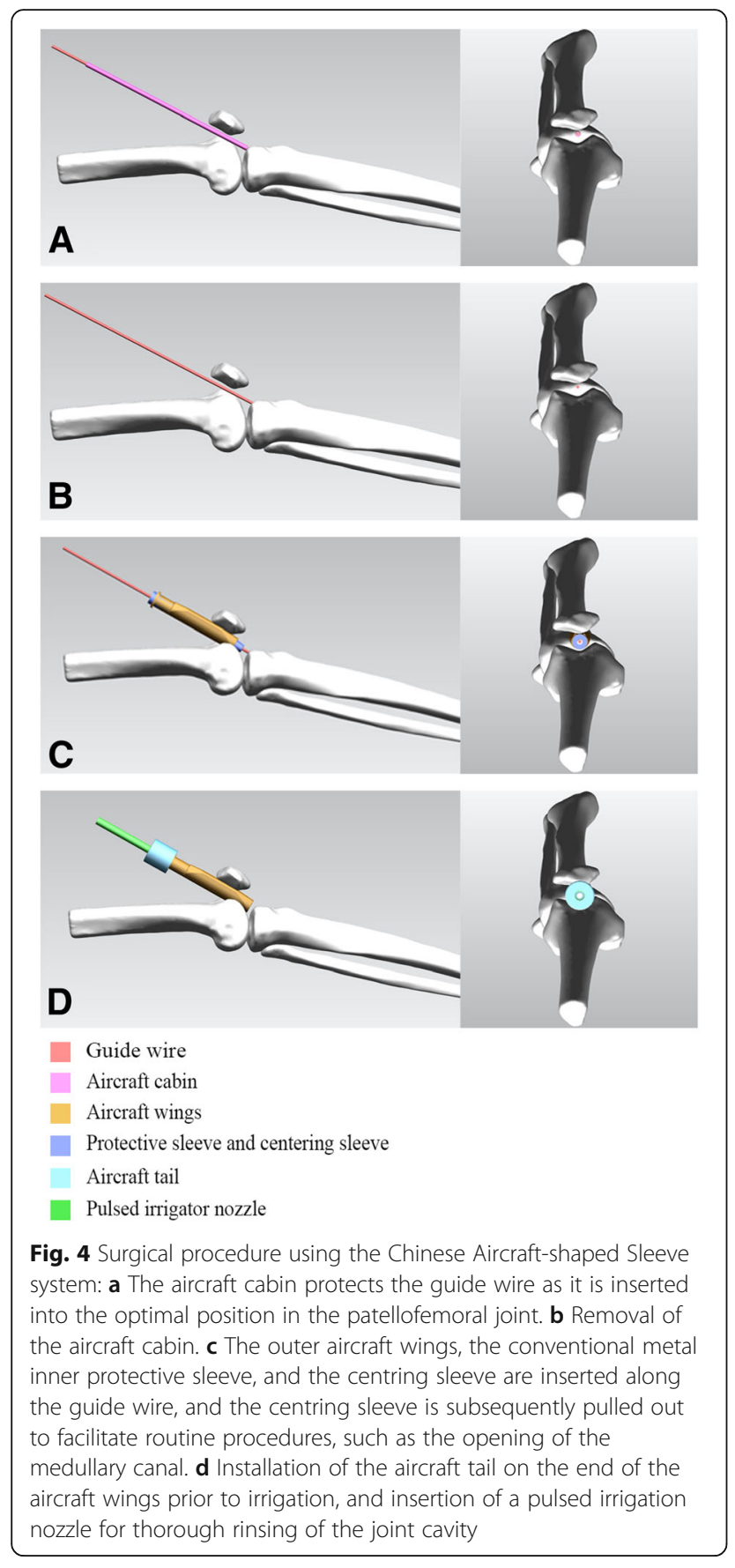

shape for inserting a conventional metal circular inner sleeve. The outer layer is $\mathrm{O}$-shaped in the axial position and extends on both sides to become V-shaped, thus resembling aircraft wings, which further stabilise the sleeve, such that even if high pressure is applied to the patellofemoral joint, the sleeve will not slide relative to the patella. Similar to the conventional infrapatellar approach, the entry hole of the nail was made in the direction of the guide wire after removal of the centring sleeve. Closed or open reduction was then performed. A guide wire was inserted for reaming followed by the appropriate intramedullary nail. The distal and proximal locking screws were fixed, and the end cap was inserted. After pulling out the metal guiding device, the surgical space was irrigated thoroughly. We also designed a connector (aircraft tail) for the end of the sleeve to improve flushing. The head has a cross-lock design that can be tightly joined or quickly separated by a rotation. The cross section of the tail is a hollow circle, similar in size to the pulsed irrigation nozzle (WJ-06, Wanjie Medical Device Co., Ltd., China). This section allows passage of the irrigation nozzle, with the nozzle reaching the area surrounding the opening of the tibia through the hollow tube, allowing for thorough irrigation and aspiration of the debris from the joint cavity. In this study, we used $4000 \mathrm{ml}$ of sterile saline for irrigation. The incision was then closed in layers.

The conventional sleeve group underwent surgery using traditional suprapatellar procedures for the ETN system (DePuy Synthes, Raynham, MA, USA). The incision and joint cavity were irrigated repeatedly with a solution volume of $4000 \mathrm{ml}$.

\section{Clinical outcomes}

The following outcomes were obtained for analysis: surgical time, calculated up to the point of remaining; arthroscopic assessment of the condition of the patellofemoral joint cartilage at two time points (once after deep separation, before sleeve insertion, and once after insertion of the end cap), using a Smith \& Nephew arthroscope system (Smith \& Nephew, Andover, MA, USA); the Outerbridge scale [5] which provides a grading of cartilage damage (grade 0, normal; grade I, cartilage with swelling and softening; grade II, fragmentation and fissuring in an area $\leq 0.5$ in. in diameter; grade III, fragmentation and fissuring in an area $>0.5$ in. diameter; and grade IV, erosion of the cartilage down to the bone); and the irrigation time. The number of cases with (positive) or without (negative) cartilage damage and residual debris was recorded for both groups. Arthroscopic evaluations were performed by a sports medicine expert, who did not have knowledge of group allocation.

\section{Statistics}

Statistical analysis was performed using SAS 9.2 (SAS Institute, Inc., Cary, NC, USA). The data collected for PA, SA, SW, and SD were divided into two groups by sex. A $t$ test was used to compare the sample means, adopting a completely randomised design. The Shapiro-Wilk test was used to test data normality, and the $F$ test was used to evaluate the homogeneity of variance. The chi-square test, with correction for continuity in a $2 \times 2$ table, was used to compare the cartilage 

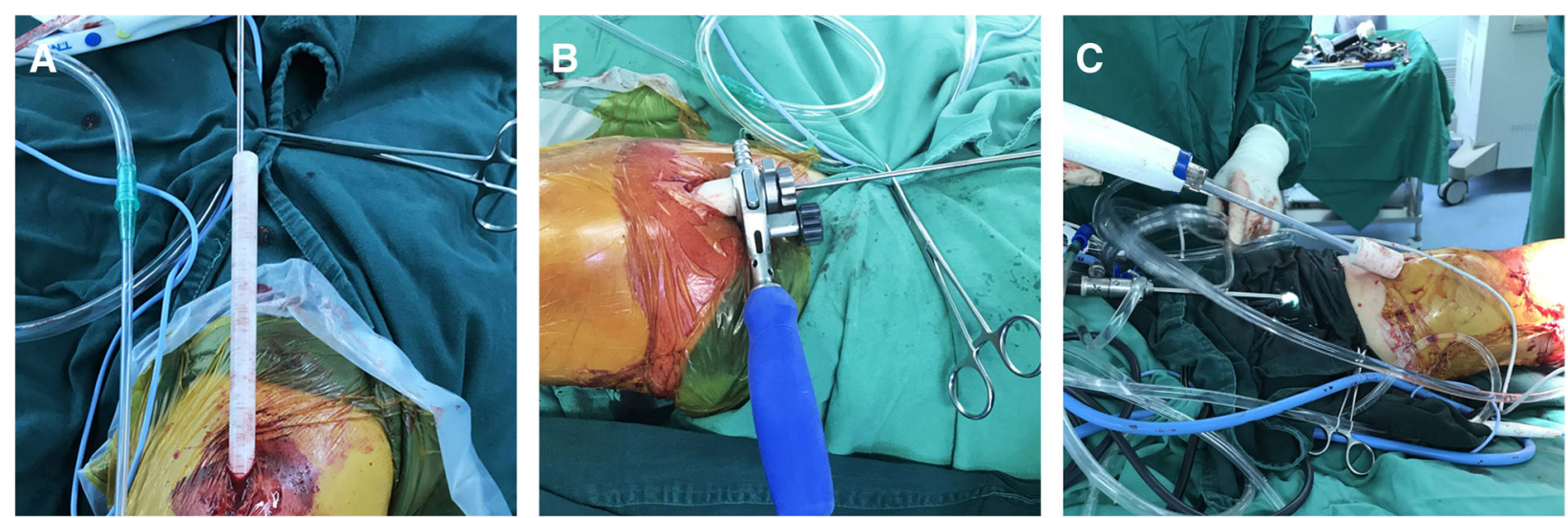

Fig. 5 Chinese Aircraft-shaped Sleeve system surgical technique: a Advanced positioning technology combining the aircraft cabin and the guide wire. $\mathbf{b}$ Reinforcement and stabilisation of the aircraft wings. $\mathbf{c}$ Rinsing technique, combining the aircraft tail and pulsed irrigation

damage rate and the residual rate of debris between the groups. $P<0.05$ indicated statistical significance.

\section{Results}

The mean \pm standard deviation of SA was $142.37 \pm 5.33^{\circ}$, with no statistically significant difference between sexes $(P=0.513)$. The mean \pm standard deviation for PA was $135.40 \pm 6.20^{\circ}$, SW was $33.37 \pm 2.73 \mathrm{~mm}$, and SD was $4.29 \pm 0.63 \mathrm{~mm}$, with a significant between-sex difference $(P<0.001$; Table 1$)$.

In the CASS group, the surgical time until entry reaming commenced in the CASS group was $5.9 \pm 1.5 \mathrm{~min}$, compared to $9.8 \pm 2.0 \mathrm{~min}$ in the conventional sleeve group, which was a significant difference $(P<0.001$; Fig. 6a). The CASS group irrigation time was $6.9 \pm 0.8 \mathrm{~min}$, whereas it was $16.7 \pm 0.9 \mathrm{~min}$ for the conventional sleeve group. The use of the aircraft tail and the pulsed irrigation device saved an average of $9.8 \mathrm{~min}$ of operative time, and the difference was significant $(P<0.001 ;$ Fig. $6 \mathrm{~b})$.

In the CASS group, the patellofemoral joint cartilage of 29 patients was not damaged postoperatively (Fig. 7); only one patient experienced cartilage damage. In the conventional sleeve group, postoperative patellofemoral joint cartilage damage was found in eight patients. Comparing the cartilage damage rate between both groups revealed $P=0.0301$; thus, the difference was statistically significant (Table 2). During arthroscopic assessment, we also measured the pressure exerted on the protective sleeve inside the patellofemoral joint in the absence of a polyetheretherketone (PEEK) trocar or metal inner protective sleeve (Fig. 8). Two of the cases of articular damage in the conventional sleeve group were caused by damage to the sleeve (Fig. 9), resulting in a change in the Outerbridge classification from a grade of "0," preoperatively, to a grade III, postoperatively.

On arthroscopic assessment, residual debris was identified, after irrigation, in one case in the CASS group compared to five cases in the conventional sleeve group $(P=0.197$, calculated using the chi-square test with correction for continuity in a $2 \times 2$ table; Table 3 ).

\section{Discussion}

The suprapatellar approach is initiated through the patellofemoral joint gap. As such, the relatively smaller body size of Chinese individuals, compared to Westerners, reduces the joint space available for manoeuvring and insertion of the intramedullary nail. It is to address this specific issue of size difference between two ethnic populations that we developed the CASS based on reliable anatomical parameters of the patellofemoral joint measured in a sample group of 212 individuals using MRI. MRI was selected as it effectively displays cartilage and periarticular soft tissues [6].

Table 1 Measurements of patellofemoral joint anatomy in 212 normal adult Chinese people

\begin{tabular}{lllll}
\hline Measured parameter & Men $(n=101)$ & Women $(n=111)$ & Total & $P$ value* \\
\hline Patella angle $\left(^{\circ}\right)$ & $137.30 \pm 6.02$ & $133.68 \pm 5.87$ & $135.40 \pm 6.20$ & $<0.001$ \\
Sulcus angle $\left(^{\circ}\right)$ & $142.62 \pm 5.32$ & $142.14 \pm 5.36$ & $142.37 \pm 5.33$ & 0.5128 \\
Sulcus width $(\mathrm{mm})$ & $35.17 \pm 2.23$ & $31.72 \pm 2.01$ & $33.37 \pm 2.73$ & $<0.001$ \\
Sulcus depth $(\mathrm{mm})$ & $4.54 \pm 0.58$ & $4.06 \pm 0.58$ & $4.29 \pm 0.63$ & $<0.001$ \\
\hline
\end{tabular}



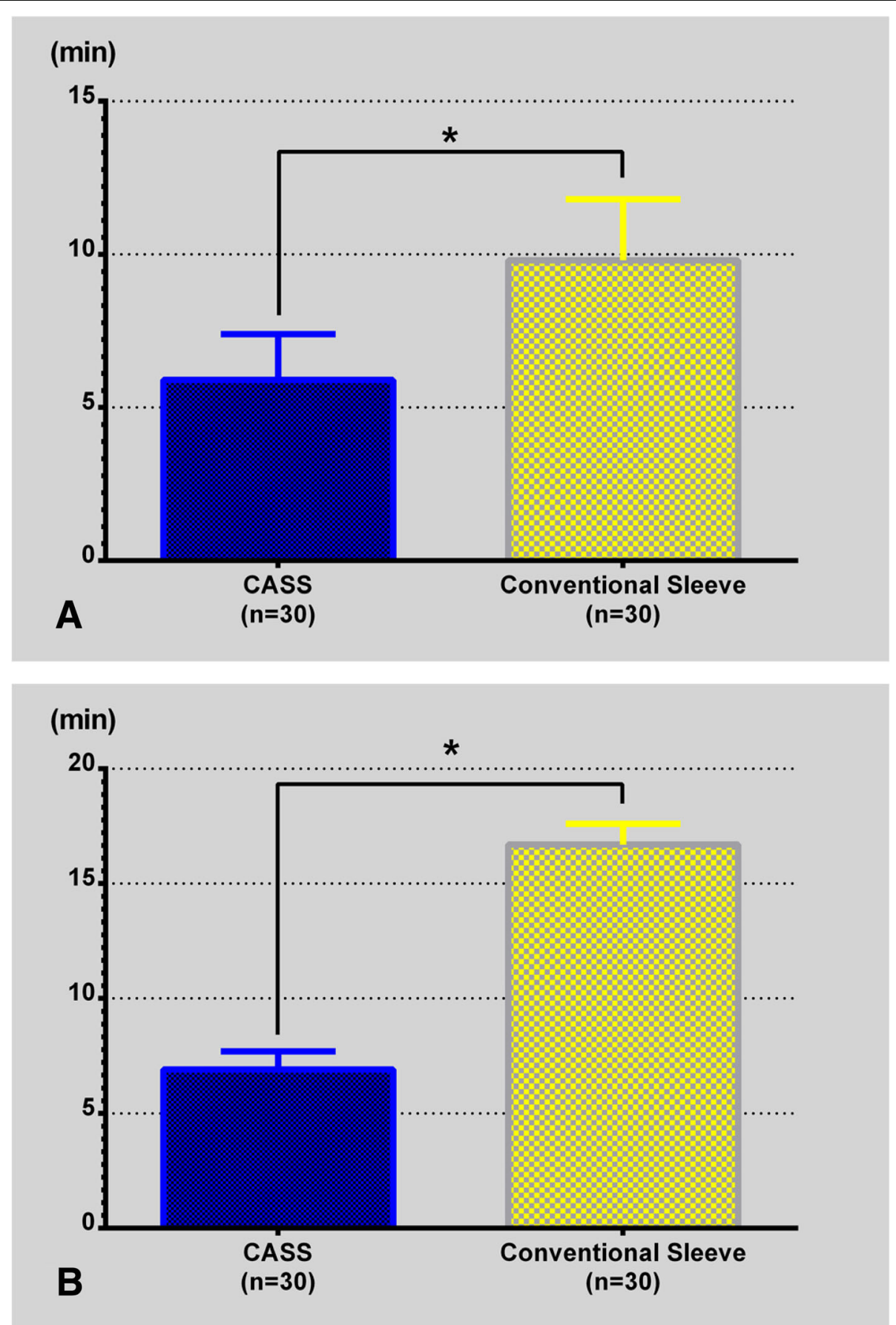

Fig. 6 Comparison of surgical times: a Surgical time until entry reaming commencement. b Irrigation time. Overall, use of the CASS system shortens the surgical time. CASS: Chinese Aircraft-shaped Sleeve system, ${ }^{*} P<0.0001$

MRI also provides images in multiple views, which facilitates observation of the patellofemoral joint from various angles, accurately displaying the area of contact between the patella and the femoral sulcus [7]. It has been suggested that the sulcus angle is consistently smaller when measured from subchondral bone than from cartilage $\left(5-7^{\circ}\right)$. Moreover, MRI is the most suitable tool for longitudinal studies of the femoral sulcus [8]. Therefore, the MRIs of the knee joints of 212 healthy Chinese people were evaluated; measurements among women were significantly smaller than those among men $(P<0.05)$. 

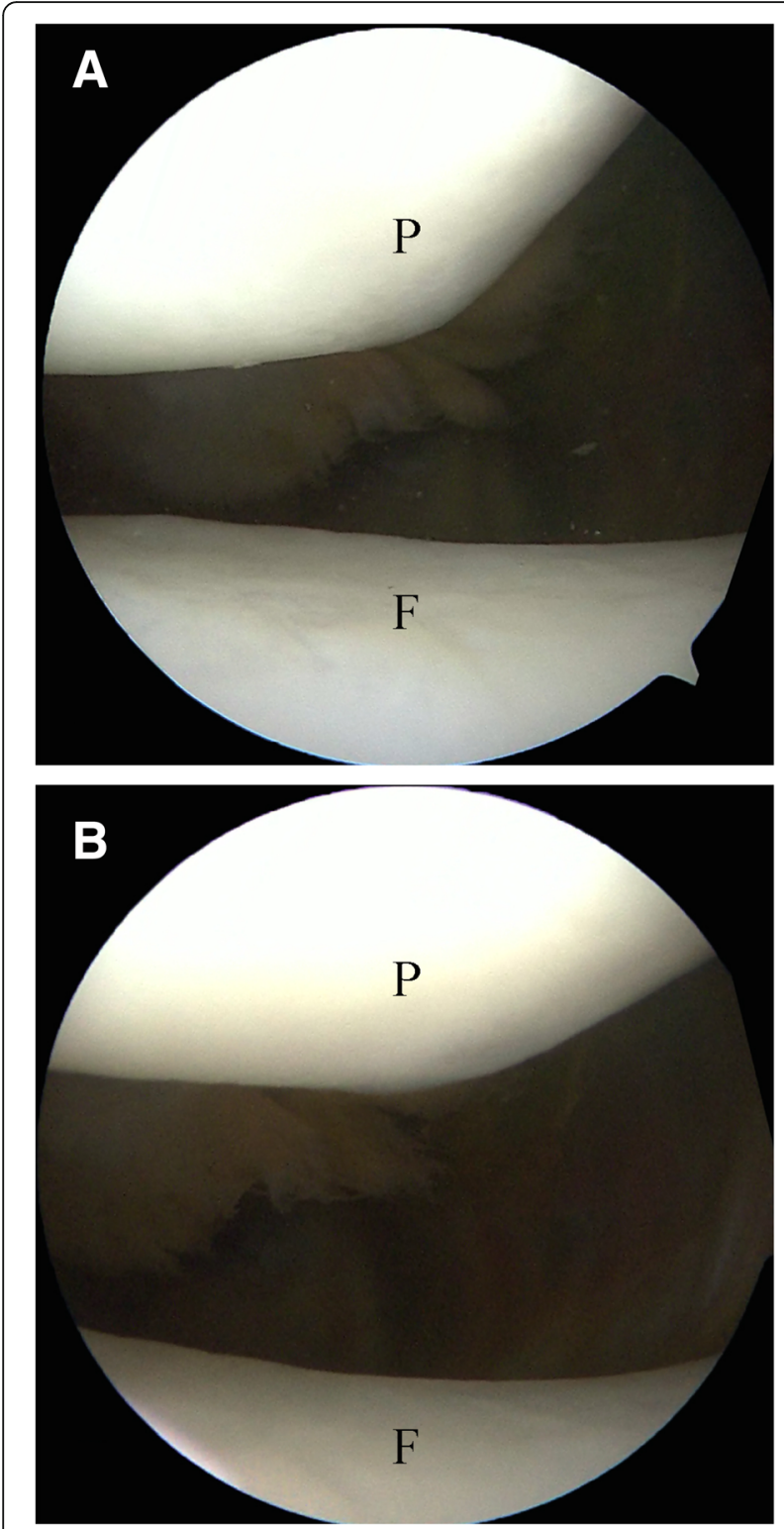

Fig. 7 Arthroscopic assessment: a Preoperative Outerbridge scale: normal. b Postoperative Outerbridge scale: normal

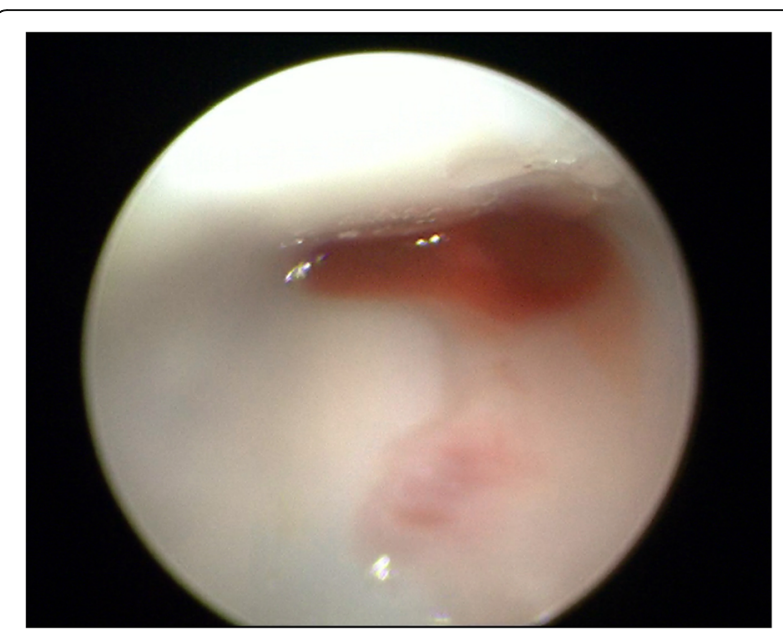

Fig. 8 Image showing that the protective sleeve is pressed into an oblate circle in the patellofemoral joint in the absence of a PEEK trocar or metal inner protective sleeve

Although soft tissue has a certain degree of elasticity and the patella is lifted after incising the quadriceps, it is difficult to insert a protective sleeve into the narrow patellofemoral joints of Chinese surgical patients, and this is especially true of Chinese women, which increases operative time. Moreover, we observed greater pressure being exerted on the protective sleeve inside the patellofemoral joint (Fig. 8). Careless or forceful insertion of an intramedullary nail through a deformed sleeve increases the risk for iatrogenic injury. In our study, two patients in the conventional sleeve group experienced articular cartilage damage because of damaged sleeves (Fig. 9). Yasuda et al. [9] proposed an improved parapatellar approach for the narrow patellofemoral joint gap. This approach includes a 5-6-cm skin incision made on the lateral side of the patella and slitting of the lateral patellar retinaculum until the joint capsule can be opened, with a 2-0 absorbable monofilament suture used to achieve and maintain patellar eversion. Following this alternate procedure, conventional tibial reaming and IMN were performed. However, this approach to increasing the surgical workspace requires cutting the retinaculum surrounding the patella. In a cadaver study, Sandmeier et al. [10] compared the patellar tracking status of intact,

Table 2 Comparison of cartilage damage rate between 30 patients in the Chinese Aircraft-shaped Sleeve group and 30 patients in the conventional sleeve group

\begin{tabular}{|c|c|c|c|c|c|}
\hline Group & Positive & Negative & Total & Residual rate (\%) & $P$ value* \\
\hline $\begin{array}{l}\text { Chinese Aircraft-shaped } \\
\text { Sleeve group }\end{array}$ & 1 & 29 & 30 & 3.33 & 0.0301 \\
\hline $\begin{array}{l}\text { Conventional } \\
\text { sleeve group }\end{array}$ & 8 & 22 & 30 & 26.67 & \\
\hline Total & 9 & 51 & 60 & 15.00 & \\
\hline
\end{tabular}

*Chi-square test with correction for continuity in a $2 \times 2$ table 

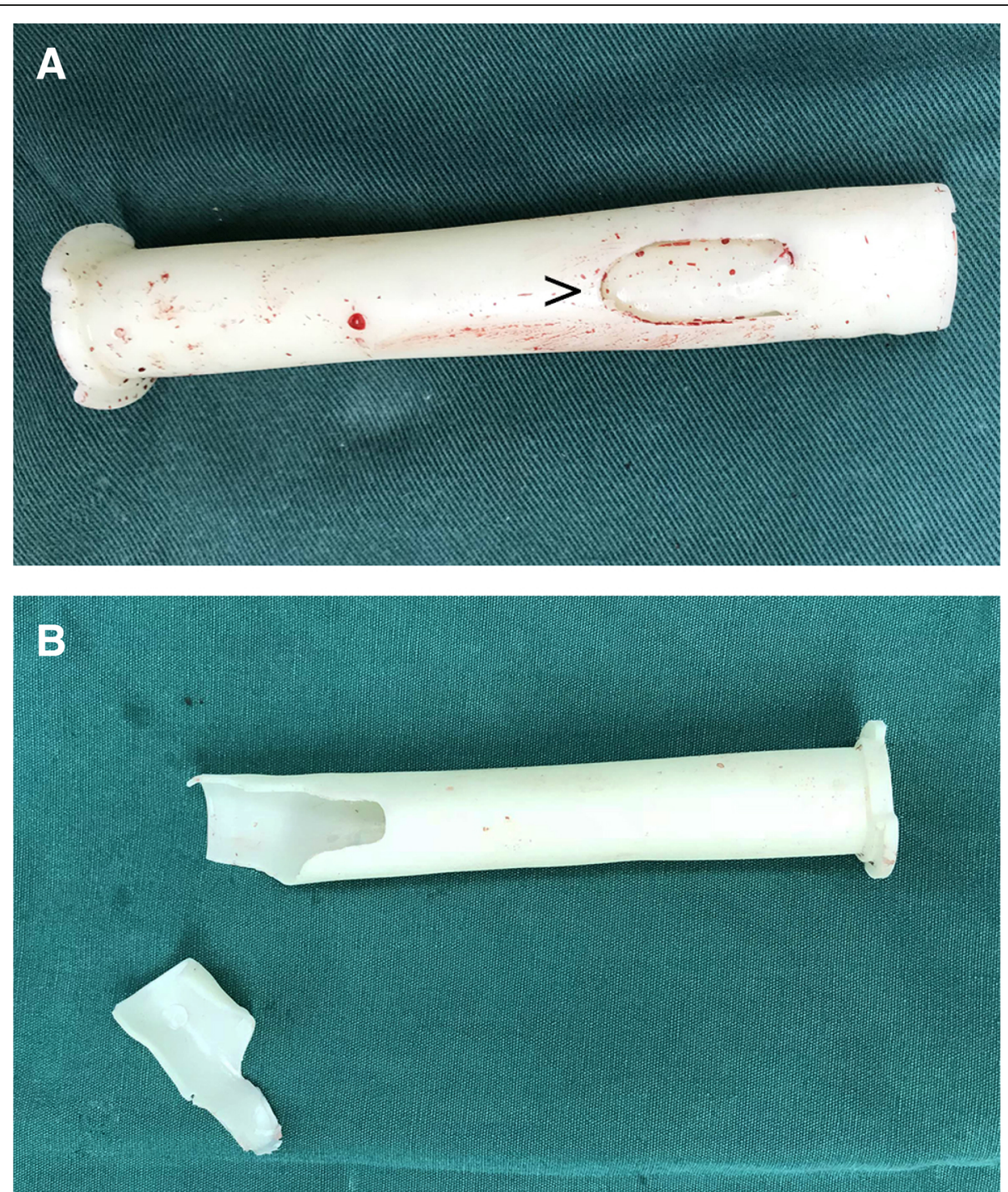

Fig. 9 Two cases ( $\mathbf{a}$ and $\mathbf{b}$ ) of sleeve damage in the conventional sleeve group; ">" indicates a crack in the sleeve

incised, and reconstructed medial patellar retinacula. With the application of a lateral stress, patellar tracking changed significantly after medial retinaculum incision. After reconstruction, patellar tracking improved, but could not be restored to a completely normal state. Therefore, we believe that the suprapatellar approach to retinaculum incision for patellar subluxation requires further study.
Another difficulty that has previously been encountered during positioning is related to the use of a circular hollow sleeve to protect the patellofemoral joint in the traditional suprapatellar approach. Tajima et al. [3] designed a heart-shaped outer sleeve for the suprapatellar approach. The ventral side of the sleeve was affixed to the posterior articular surface of the patella at $130^{\circ}$. The dorsal side was affixed to the femoral sulcus at $140^{\circ}$. In our measurement

Table 3 Comparison of postoperative residual rate of debris between 30 patients in the Chinese Aircraft-shaped Sleeve group and 30 patients in the conventional sleeve group

\begin{tabular}{llllll}
\hline Group & Positive & Negative & Total & Residual rate (\%) & $P$ value* \\
\hline Chinese Aircraft-shaped Sleeve group & 1 & 29 & 30 & 3.33 & 0.1967 \\
Conventional sleeve group & 5 & 25 & 30 & 16.67 \\
Total & 6 & 54 & 60 & 10.00 \\
\hline
\end{tabular}

*Chi-square test with correction for continuity in a $2 \times 2$ table 
of anatomical parameters, we determined that these angles are inappropriate for Chinese individuals, with a PA of $135.40 \pm 6.20^{\circ}$ and $\mathrm{SA}$ of $142.37 \pm 5.33^{\circ}$ when the medial and lateral cartilage surfaces of the patella and sulcus, respectively, meet in the middle. Based on these measurements, we designed an anatomically correct protective outer sleeve (aircraft wings), with a ventral face angle of $135^{\circ}$ and the dorsal face angle of $142^{\circ}$.

Currently, some scholars are concerned that the suprapatellar approach may cause iatrogenic injuries [11-15]. In this study, cartilage damage was also observed in the conventional sleeve group (Fig. 10), and the rate was higher than that in the CASS group. It may be that the patellar gaps in persons of Chinese descent are small, leading to iatrogenic injury when sleeves or intramedullary nails are inserted. The main methods of reducing the above iatrogenic injuries include (1) using the aircraft cabin advance positioning technology; (2) retaining the protective sleeve intraoperatively; (3) preventing the knee joint from being straightened when the nail is being inserted; (4) avoiding forceful separation of the outer sleeve and the inner metal sleeve or PEEK trocar, as the outer sleeve is soft and would be deformed by pressure if it exists in isolation in a narrow patellofemoral joint; (5) stopping the reamer rotation and lifting up the drill to centre the reamer shaft in the sleeve, then moving the reamer in and out until the reamer comes out in a situation wherein the reamer is caught on the edge of the inner protection sleeve because of its sharp angle of entry into the tibia; and (6) using general anaesthesia when certain muscle relaxation is required.

The suprapatellar approach is performed through the joint cavity, and debris are generated when open reaming is performed. If debris remain in the joint cavity after the intraoperative procedures, there is a risk of heterotopic ossification or joint mice. Sanders et al. [13] reported that debris does not form new bones in synovial fluid; hence, heterotopic ossification is not a serious problem, but thorough postoperative irrigation is still necessary. In the conventional suprapatellar approach, the flow rate is limited, the direction of flow is not controllable, and blind angles are not easily irrigated. Consequently, the irrigation time tends to be long and the process may not be sufficiently thorough to avoid postoperative issues. To avoid this, it is mandatory to use a large amount of saline to perform pressurised irrigation in all directions. Currently, there are no relevant studies to indicate the amount of solution that is needed. We referred to a study by Niki et al. [16] that investigated the amount of saline needed for effective irrigation in total knee arthroplasty. Their results showed that $4000 \mathrm{ml}$ of pulsed irrigation was the most efficient method of removing bone and bone cement debris. Therefore, in this study, we also used $4000 \mathrm{ml}$ of rinsing solution. Our conventional sleeve group had a total irrigation time of $16.7 \pm 0.9 \mathrm{~min}$, which was considered a long time. It was clear that in routine surgery, manual irrigation using $4000 \mathrm{ml}$ of saline would be too time-consuming and laborious. Operating the aircraft tail combined with the pulsed irrigation is much simpler. Pressure causes the fluid to flow at a certain frequency and shooting force. The debris and bacteria in the joint cavity are separated from the tissue by the oscillating action of the pulsed water flow. During irrigation, the irrigation solution is drawn out through the aspiration channel. The total irrigation time in the CASS group was $6.9 \pm 0.8 \mathrm{~min}$, which was greatly reduced compared to that of the conventional group. Furthermore, the more irrigation solution used, the greater the reduction of irrigation time.

This study had several limitations, including the small sample size and insufficient postoperative follow-up time. In the future, a randomised controlled study based on a larger sample should be performed. Additionally, $4 \mathrm{~L}$ irrigation would be too time-consuming and laborious in routine surgery. We would recommend that 1-2 L of irrigation fluid would be more suitable for efficient removal of bone debris during tibial IMN. The most appropriate amount of irrigation needs to be determined in further studies.
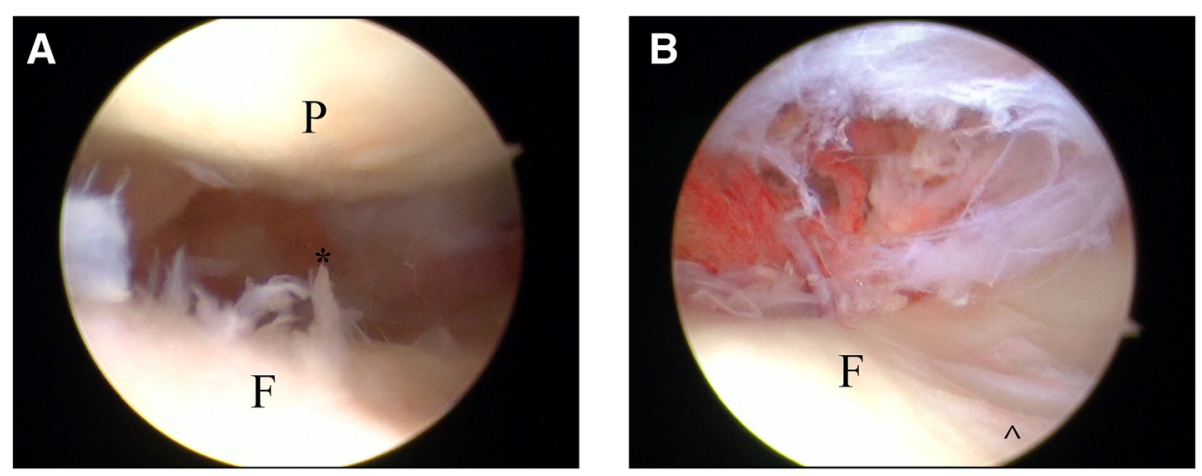

Fig. 10 Cartilage injury observed by arthroscopy in the conventional sleeve group. a "**" Cartilage fragmentation and fissuring. $\mathbf{b}$ " $\wedge "$ Cartilage displays injury in the shape of a ski trail 


\section{Conclusion}

This study demonstrated that the CASS system provides several advantages, including simplicity in the positioning of the protective sleeve, stability of the sleeve after insertion, minimal articular cartilage damage, convenient intraoperative irrigation, and shorter surgical times. This novel and practical improvement may bring about beneficial outcomes for tibial shaft fractures using the suprapatellar approach during intramedullary nailing, especially for the treatment of smaller patients with tibial shaft fractures.

\section{Abbreviations}

CASS: Chinese Aircraft-shaped Sleeve; IMN: Intramedullary nailing; MRI: Magnetic resonance image; PA: Patellar angle; PEEK: Polyetheretherketone; SA: Sulcus angle; SD: Sulcus depth; SW: Sulcus width; TU: Thermoplastic polyurethane

\section{Acknowledgements}

Not applicable.

\section{Funding}

Not applicable.

\section{Availability of data and materials}

The datasets used and/or analysed during the current study are available from the corresponding author on reasonable request.

\section{Authors' contributions}

$\mathrm{KL}, T \mathrm{Z}, \mathrm{YJG}, \mathrm{HZW}$, and QRD contributed to the study conception and design. TTZ and YW contributed to the acquisition of data. ZQW and TTZ contributed to the analysis and interpretation of data. DGZ contributed the design and fabrication of the CASS system. $\mathrm{KL}$ drafted the manuscript, and $\mathrm{QRD}$ revised it. All authors read and approved the final manuscript.

Ethics approval and consent to participate

This study has been approved by the Ethics Committee of the First People's Hospital of Kunshan (NO. 20150512). Written consent was obtained from all participants in the study.

\section{Consent for publication}

Not applicable.

\section{Competing interests}

The authors declare that they have no competing interests.

\section{Publisher's Note}

Springer Nature remains neutral with regard to jurisdictional claims in published maps and institutional affiliations.

\section{Author details}

${ }^{1}$ Department of Orthopaedics, the Second Affiliated Hospital of Soochow University, 1055 Sanxiang Road, Suzhou 215004, Jiangsu, China. ${ }^{2}$ Department of Joint Surgery, the First People's Hospital of Kunshan, Suzhou 215300, Jiangsu, China. ${ }^{3}$ Department of Radiology, the First People's Hospital of Kunshan, Suzhou 215300, Jiangsu, China. ${ }^{4}$ R\&D Department, Jiangsu OKANI Medical Technology Co., Lt, Suzhou 215214, Jiangsu, China.

Received: 4 October 2018 Accepted: 2 November 2018

Published online: 14 November 2018

\section{References}

1. Kuhn S, Greenfield J, Arand C, Jarmolaew A, Appelmann P, Mehler D, et al. Treatment of distal intraarticular tibial fractures: A biomechanical evaluation of intramedullary nailing vs. angle-stable plate osteosynthesis. Injury. 2015; 46(Suppl 4):99-103.

2. Tornetta P 3rd, Collins E. Semiextended position of intramedullary nailing of the proximal tibia. Clin Orthop Relat Res. 1996;328:185-9.
3. Tajima K, Shimizu C, Ohno S, Nishida Y, Udagawa K, Sasaki JA. Heart-shaped sleeve simplifies intramedullary tibial nail insertion when using the suprapatellar approach. Keio J Med. 2018;67(1):10-6.

4. Bacchetti $P$, Leung JM. Sample size calculations in clinical research. Anesthesiology. 2002;97(4):1028-9 author reply 9-32.

5. Vellet AD, Marks PH, Fowler PJ, Munro TG. Occult posttraumatic osteochondral lesions of the knee: prevalence, classification, and short-term sequelae evaluated with MR imaging. Radiology. 1991;178(1):271.

6. Mcgibbon CA, Trahan CA. Measurement accuracy of focal cartilage defects from MRI and correlation of MRI graded lesions with histology: a preliminary study. Osteoarthr Cartil. 2003;11(7):483-93.

7. Eisenhart-Rothe Rv SM, Bringmann C, Vogl T, Englmeier K-H, Graichen H. A new in vivo technique for determination of $3 \mathrm{D}$ kinematics and contact areas of the patello-femoral and tibio-femoral joint. J Biomech. 2004;37(6):927-34.

8. Toms A, Cahir J, Swift L, Donell S. Imaging the femoral sulcus with ultrasound, CT, and MRI: reliability and generalizability in patients with patellar instability. Skelet Radiol. 2009;38(4):329-38.

9. Yasuda T, Obara S, Hayashi J, Arai M, Sato K. Semiextended approach for intramedullary nailing via a patellar eversion technique for tibial-shaft fractures: evaluation of the patellofemoral joint. Injury. 2017;48(6):1264-8.

10. Sandmeier RH, Burks RT, Bachus KN, Billings A. The effect of reconstruction of the medial patellofemoral ligament on patellar tracking. Am J Sports Med. 2000;28(3):345.

11. Zelle BA. Intramedullary nailing of tibial shaft fractures in the semi-extended position using a suprapatellar portal technique. Int Orthop. 2017;41(9):1909-14.

12. Eastman J, Tseng S, Lo E, Li CS, Yoo B, Lee M. Retropatellar technique for intramedullary nailing of proximal tibia fractures: a cadaveric assessment. J Orthop Trauma. 2010;24(11):672-6.

13. Sanders RW, Dipasquale TG, Jordan CJ, Arrington JA, Sagi HC. Semiextended intramedullary nailing of the tibia using a suprapatellar approach: radiographic results and clinical outcomes at a minimum of 12 months follow-up. J Orthop Trauma. 2014;28(5):245.

14. Chan DS, Serrano R, Griffing R, Steverson B, Infante A, Watson D, et al. Supra- versus infra-patellar tibial nail insertion: a prospective, randomized control pilot study. J Orthop Trauma. 2015;30(3):130.

15. Gelbke MK, Coombs D, Powell S, Dipasquale TG. Suprapatellar versus infrapatellar intramedullary nail insertion of the tibia: a cadaveric model for comparison of patellofemoral contact pressures and forces. J Orthop Trauma. 2010;24(11):665-71.

16. Niki Y, Matsumoto H, Otani T, Tomatsu T, Toyama Y. How much sterile saline should be used for efficient lavage during total knee arthroplasty? Effects of pulse lavage irrigation on removal of bone and cement debris. J Arthroplasty. 2007;22(1):95-9.

\section{Ready to submit your research? Choose BMC and benefit from:}

- fast, convenient online submission

- thorough peer review by experienced researchers in your field

- rapid publication on acceptance

- support for research data, including large and complex data types

- gold Open Access which fosters wider collaboration and increased citations

- maximum visibility for your research: over $100 \mathrm{M}$ website views per year

At BMC, research is always in progress.

Learn more biomedcentral.com/submission 